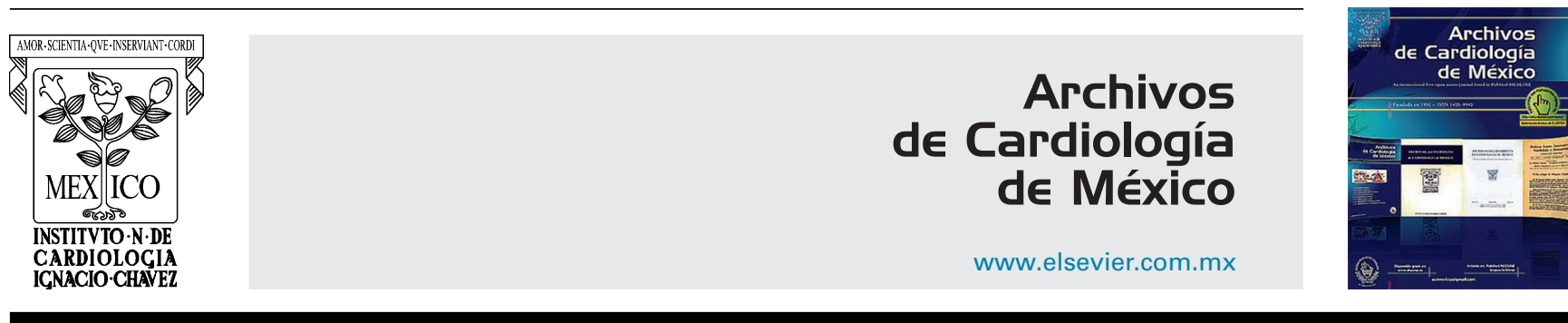

INVESTIGACIÓN CLÍNICA

\title{
Síndrome de Bayés en cirugía cardiaca: prevalencia de bloqueo interauricular en menores de 65 años sometidos a cirugía cardiaca y su asociación con fibrilación auricular en el postoperatorio
}

\author{
Eusebio García-Izquierdo Jaén*, Pablo Cobo Rodríguez, Luis Solís Solís, \\ Chinh Pham Trung, Diego Jiménez Sánchez, Manuel Sánchez García, \\ Victor Castro Urda, Jorge Toquero Ramos e Ignacio Fernández Lozano
}

Unidad de Arritmias, Hospital Universitario Puerta de Hierro, Majadahonda, Madrid, España

Recibido el 16 de mayo de 2017; aceptado el 27 de septiembre de 2017

\section{PALABRAS CLAVE \\ Fibrilación auricular; Fibrilación auricular postoperatoria; Cirugía cardiaca; Bloqueo interauricular; Síndrome de Bayés; España}

\begin{abstract}
Resumen
Objetivo: El bloqueo interauricular (BIA) es una entidad asociada con un mayor riesgo de presentar fibrilación auricular (FA), constituyendo el denominado síndrome de Bayés. El objetivo de nuestro estudio fue definir la prevalencia de BIA en pacientes menores de 65 años sometidos a cirugía cardiaca y determinar si existe una asociación entre la presencia de BIA y la aparición de FA postoperatoria.

Método: Se incluyeron un total de 207 pacientes. Se definió BIA parcial como onda $\mathrm{P}>120 \mathrm{~ms}$. Se definió $\mathrm{BIA}$ avanzado como onda $\mathrm{P}>120 \mathrm{~ms}$ con morfología bifásica en derivaciones inferiores. Se determinó la frecuencia de aparición de FA postoperatoria y se realizó un análisis comparativo entre los pacientes que presentaron y los que no presentaron esta arritmia.

Resultados: La prevalencia de BIA fue del 78.3\% (parcial, 66.2\%; avanzado, 12.1\%). La frecuencia de aparición de FA fue del $28.5 \%$ de forma global, siendo mayor en los pacientes con BIA avanzado (44\%) comparado con pacientes con BIA parcial $(27.7 \%$ ) y sin BIA (24.4\%). Los pacientes que presentaron FA fueron significativamente mayores, con niveles más elevados de NTproBNP y presentaron mayor prevalencia de dilatación auricular y de patología tiroidea. El análisis multivariable demostró una asociación independiente entre BIA avanzado y FA postoperatoria.
\end{abstract}

\footnotetext{
* Autor para correspondencia. Hospital Universitario Puerta de Hierro. Servicio de Cardiología. Manuel de Falla, 1. 28222. Majadahonda. Madrid (España). Teléfono: +34 911916000.

Correo electrónico: usegij@gmail.com (E. García-Izquierdo Jaén).
} 
Conclusiones: El BIA es una entidad frecuente en pacientes sometidos a cirugía cardiaca. En nuestro estudio, el BIA avanzado se asocia de forma independiente con una mayor frecuencia de FA postoperatoria en pacientes menores de 65 años sometidos a cirugía cardiaca.

(C) 2017 Instituto Nacional de Cardiología Ignacio Chávez. Publicado por Masson Doyma México S.A. Este es un artículo Open Access bajo la licencia CC BY-NC-ND (http://creativecommons. org/licenses/by-nc-nd/4.0/).

\section{KEYWORDS}

Atrial fibrillation; Postoperative atrial fibrillation; Cardiac surgery; Interatrial block; Bayes' syndrome; Spain

\section{Introducción}

El bloqueo interauricular (BIA) consiste en un retraso en la conducción del estímulo eléctrico entre ambas aurículas. Este retraso de la conducción tiene lugar a nivel de unas fibras musculares que constituyen el haz de Bachmann y que conectan ambas aurículas en su parte superior ${ }^{1}$. El primer caso de BIA fue descrito por primera vez por George Bachmann en $1941^{2}$. Sin embargo, no fue hasta el año $1979^{3}$ cuando Bayés de Luna definió dos tipos de bloqueo en función de los hallazgos electrocardiográficos: el bloqueo interauricular parcial (BIA-P), que es aquel en el que se evidencia una onda $P$ de más de $120 \mathrm{~ms}$ de duración en cualquiera de las derivaciones, y el bloqueo interauricular avanzado (BIA-AV), que es aquel en el que además se objetiva una morfología bifásica de la onda $\mathrm{P}$ (con componente positivo-negativo) en alguna de las derivaciones de la cara inferior (II, III O aVF). Este trastorno de la conducción a nivel auricular ha sido ampliamente estudiado en las últimas décadas y se ha relacionado su presencia con la aparición de taquiarritmias auriculares, en concreto fibrilación auricular (FA) y flutter auricular. Esta asociación, descrita por primera vez en $1988^{4}$, constituye un síndrome denominado síndrome de Bayés ${ }^{5,6}$. Por otro lado, también se ha sugerido en la literatura una posible relación entre la presencia de BIA y el riesgo de presentar eventos tromboembólicos, incluso en ausencia de FA o flutter auricular ${ }^{7-10}$.

$\mathrm{El}$ síndrome de Bayés ha sido estudiado en múltiples escenarios. Datos de nuestro grupo de trabajo objetivaron una elevada prevalencia de BIA en pacientes de edad avanzada sometidos a cirugía cardiaca, con una frecuencia de aparición de FA en estos pacientes que duplicaba a la de aquellos que no presentaban $\mathrm{BI}^{11}$. Sin embargo, no fue posible demostrar en esta población una asociación independiente entre BIA y FA postoperatoria, probablemente por ser la edad un factor de confusión. Por ello, el objetivo del presente estudio ha sido analizar la prevalencia de BIA-P y BIA-AV en una población más joven (menores de 65 años) y su posible asociación con la aparición de FA en el periodo postoperatorio.

\section{Métodos}

Se trata de un estudio observacional en el que se incluyeron pacientes sometidos a distintos tipos de cirugía cardiaca en 


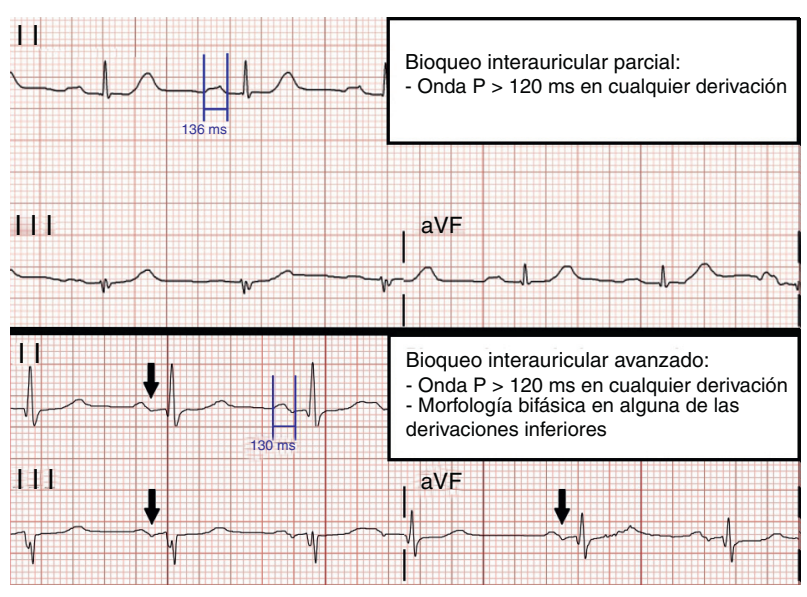

Figura 1 Ejemplo de bloqueo interauricular parcial y de bloqueo interauricular avanzado.

nuestro centro y en el que los datos fueron recogidos de forma prospectiva.

Tras revisar su evolución durante el ingreso, se determinó si habían presentado o no episodios de FA postoperatoria documentada mediante registro electrocardiográfico, desde que finalizó la cirugía hasta el alta hospitalaria. Se consideró FA postoperatoria aquellos episodios de al menos $5 \mathrm{~min}$ de duración incluyendo a quienes precisaron cardioversión urgente por inestabilidad hemodinámica. Se excluyó del análisis a todos aquellos pacientes que fallecieron durante la intervención quirúrgica.

Se midió la duración de la onda $\mathrm{P}$ de manera manual sobre el ECG de 12 derivaciones obtenido antes de la cirugía, utilizando el sistema de gestión y análisis de ECG TraceMasterVue (Phillips). Se recogió también su morfología en derivaciones inferiores. Estos datos fueron recogidos por un primer investigador (P.C.R.) y revisados posteriormente por un segundo investigador (E.G-I.J.). Toda esta información permitió clasificar a los pacientes en tres categorías: ausencia de BIA, BIA-P y BIA-AV (fig. 1).

Se analizaron datos demográficos (edad y sexo), clínicos (peso, talla, índice de masa corporal, hipertensión arterial, diabetes mellitus, dislipidemia, hábito tabáquico, enfermedad pulmonar obstructiva crónica, antecedente de patología tiroidea), parámetros ecocardiográficos (fracción de eyección de ventrículo izquierdo, volumen de aurícula izquierda), analíticos (NTproBNP antes de la cirugía) y tiempos relacionados con la cirugía (tiempo de isquemia, tiempo total de la cirugía). Se consideró el tipo de cirugía realizada para determinar la frecuencia de aparición de FA postoperatoria.

Las variables cualitativas fueron expresadas como porcentajes y las variables continuas fueron expresadas como medias (desviación estándar como medida de dispersión) y medianas (intervalo intercuartílico como medida de dispersión). Se utilizó la prueba Chi-cuadrado para comparar variables cualitativas y la pruebat de Student para comparar las medias de las muestras independientes. A fin de comparar las medianas de variables continuas que no seguían una distribución normal (NTproBNP) se utilizó la prueba $U$ de Mann-Whitney. Para llevar a cabo el análisis multivariable se empleó un modelo de regresión logística binaria, incluyendo en el modelo las variables que obtuvieron significación estadística en la comparación univariable y las que podrían tener una relación clínica relevante.

Se utilizó el paquete de datos SPSS 23 (SPSS, Inc.; Chicago, Illinois, Estados Unidos) para llevar a cabo todo el análisis estadístico. Se consideró significativo un valor de $\mathrm{p}<0.05$.

\section{Resultados}

Se incluyeron inicialmente un total de 898 pacientes sometidos a cirugía cardiaca entre enero de 2013 y septiembre de 2015. Se excluyeron aquellos pacientes que presentaban FA permanente, ritmo estimulado por marcapasos de base, pacientes sometidos a trasplante cardiaco, aquellos que fallecieron durante el procedimiento quirúrgico o en los que la onda P no era valorable en el ECG de superficie, quedando un total de 465 pacientes. Finalmente, se seleccionaron 207 pacientes cuya edad era menor de 65 años. Todos ellos contaban con un ECG previo a la cirugía en ritmo sinusal que permitió realizar el análisis de la onda $\mathrm{P}$.

El tipo de cirugía más frecuente fue la cirugía valvular aislada, seguida de la cirugía de revascularización aislada, otros tipos de cirugía y, por último, la cirugía combinada valvular y de revascularización (fig. 2).

En forma global, se diagnosticó FA en el postoperatorio en 59 pacientes (28.5\%). Se encontraron diferencias significativas en lo que respecta a la edad (mayor edad en pacientes que presentaron FA), patología tiroidea y dilatación de la aurícula izquierda (ambas más frecuentes en pacientes que presentaron FA). Se objetivaron también niveles significativamente más elevados de NTproBNP antes de la cirugía en pacientes que presentaron FA. No existieron diferencias significativas en lo que respecta a la incidencia de FA postoperatoria para el resto de variables, incluido el tiempo de isquemia y el tiempo total de cirugía. Los distintos tipos de cirugía se distribuyeron de forma homogénea en función del diagnóstico de FA postoperatoria, excepto el grupo de pacientes sometidos a cirugía de revascularización aislada. Este grupo se asoció de forma estadísticamente significativa con una menor frecuencia de aparición de FA (tabla 1).

Se identificó BIA-P en 137 pacientes (66.2\%) y BIA-AV en 25 pacientes $(12.1 \%)$. El $24.4 \%$ de los pacientes sin BIA y el $27.7 \%$ de los pacientes con BIA-P presentaron FA en el postoperatorio. En el caso de los pacientes con BIA-AV, la presencia de FA fue más frecuente $(44 \%)$, rozando esta diferencia la significación estadística $(p=0.067)$ (fig. 3).

Se investigó también cuál era la distribución del BIA-AV en función de la presencia de crecimiento auricular significativo. Se evidenció que el $8.8 \%$ de los pacientes sin dilatación auricular presentaban BIA-AV frente al $20 \%$ de los pacientes con dilatación moderada o severa de la aurícula izquierda, siendo esta diferencia estadísticamente significativa $(\mathrm{p}=0.025)$.

Con todos estos datos, se realizó un análisis multivariable, mediante regresión logística binaria, asociándose de forma independiente con la aparición de FA postoperatoria la edad, la dilatación auricular y la presencia de BIA-AV (tabla 2). 


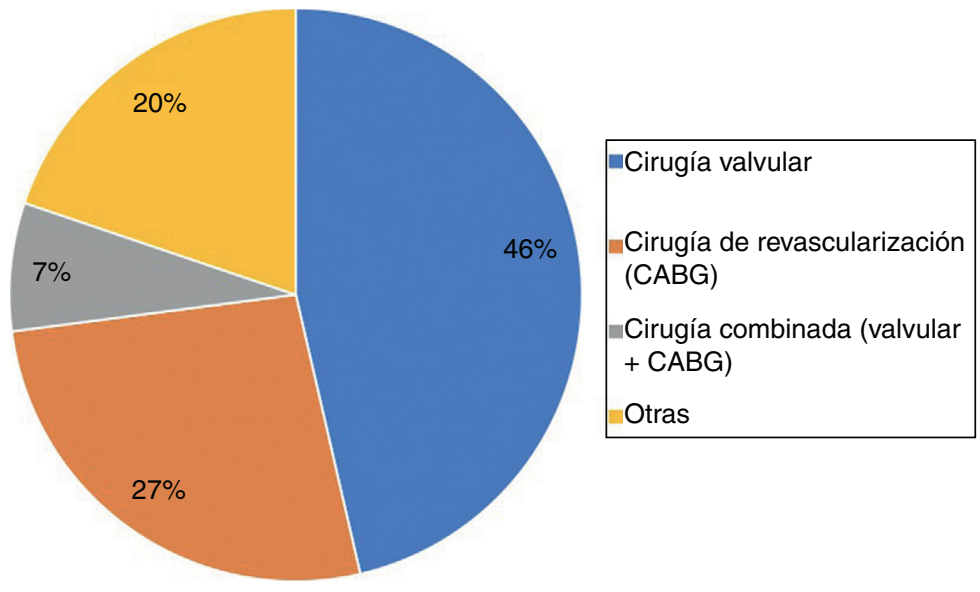

Figura 2 Se muestra la distribución de la población del estudio según los distintos tipos de cirugía. En otros tipos de cirugía se incluyen implantes de asistencias mecánicas, miomectomías y cirugías de aorta sin intervención valvular.

Tabla 1 Características de la población general y diferencias entre el grupo que presentó FA postoperatoria y el grupo que no presentó FA postoperatoria

\begin{tabular}{|c|c|c|c|c|}
\hline & Total $(n=207)$ & FA sí $(n=59)$ & FA no $(n=148)$ & $\mathrm{p}$ \\
\hline Sexo mujer & $66(31.9 \%)$ & $20(33.9 \%)$ & $46(31.1 \%)$ & 0.695 \\
\hline Edad & $53.1 \pm 9.8$ & $55.7 \pm 7.9$ & $52.1 \pm 10.3$ & 0.017 \\
\hline IMC & $26.8 \pm 4.6$ & $26.8 \pm 4.1$ & $26.9 \pm 4.8$ & 0.961 \\
\hline HTA & $89(43.0 \%)$ & $23(39.0 \%)$ & $66(44.6 \%)$ & 0.462 \\
\hline DM & $40(19.3 \%)$ & $13(22.0 \%)$ & $27(18.2 \%)$ & 0.533 \\
\hline $\mathrm{DL}$ & $80(38.6 \%)$ & $20(33.9 \%)$ & $60(40.5 \%)$ & 0.376 \\
\hline EPOC & $11(5.3 \%)$ & $5(8.5 \%)$ & $6(4.1 \%)$ & 0.201 \\
\hline Fumador & $108(52.2 \%)$ & $30(50.8 \%)$ & $78(52.7 \%)$ & 0.809 \\
\hline Enfermedad tiroidea & $13(6.3 \%)$ & $7(11.9 \%)$ & $6(4.1 \%)$ & 0.037 \\
\hline FEVI (\%) & $57.6 \pm 13.6$ & $58.2 \pm 13.8$ & $57.4 \pm 13.6$ & 0.760 \\
\hline Dilatación Al moderada-severa & $32.5 \%$ & $53.4 \%$ & $23.9 \%$ & $<0.001$ \\
\hline NTproBNP (pg/ml) & $729(172 ; 1,914)$ & $1202(401 ; 2,830)$ & $592(143 ; 1,727)$ & 0.007 \\
\hline Cirugía valvular & $96(46.4 \%)$ & $33(55.9 \%)$ & $63(42.6 \%)$ & 0.083 \\
\hline CABG & $55(26.6 \%)$ & $9(15.3 \%)$ & $46(31.1 \%)$ & 0.024 \\
\hline Valvular + CABG & $15(7.2 \%)$ & $5(8.5 \%)$ & $10(6.8 \%)$ & 0.767 \\
\hline Otras cirugías & $41(19.8 \%)$ & $12(20.3 \%)$ & $29(19.6 \%)$ & 0.972 \\
\hline Tiempo de isquemia (minutos) & $84.1 \pm 41.3$ & $84.4 \pm 48.1$ & $84.0 \pm 38.4$ & 0.961 \\
\hline Tiempo de la cirugía (minutos) & $109.6 \pm 51.6$ & $112.3 \pm 58.9$ & $108.5 \pm 48.7$ & 0.664 \\
\hline
\end{tabular}

Las variables cualitativas se expresan en número absoluto y porcentaje entre paréntesis. Las variables cuantitativas se expresan como media \pm desviación estándar, excepto NTproBNP que se expresa como mediana y rango intercuartílico.

Al: aurícula izquierda; CABG: cirugía de revascularización coronaria; DL: dislipidemia; DM: diabetes mellitus; EPOC: enfermedad pulmonar obstructiva crónica; FA: fibrilación auricular; FEVI: fracción de eyección de ventrículo izquierdo; HTA: hipertensión arterial; IMC: índice de masa corporal; NTproBNP: propéptido natriurético cerebral N-terminal.

\section{Discusión}

La FA es una complicación postoperatoria frecuente de la cirugía cardiaca, con una incidencia cercana al $50 \%$ en pacientes sometidos a cirugía valvular ${ }^{12}$ e incluso superior (en torno al 60\%) en cirugía valvular combinada con revascularización coronaria ${ }^{13}$, siendo estos los tipos de cirugía que más se asocian a FA en la mayoría de las series. El mecanismo etiopatogénico subyacente no es del todo conocido, aunque se sabe que la inflamación, el estrés oxidativo y especialmente la activación simpática desencadenada en la cirugía juegan un papel importante en la aparición de taquiarritmias auriculares en el periodo postoperatorio ${ }^{14}$.
Existen múltiples factores de riesgo descritos que se relacionan con una mayor incidencia de FA postoperatoria. Entre estos factores, cabe destacar la edad avanzada como uno de los principales predictores independientes que aparece de forma invariable en casi todas las series publicadas ${ }^{13,15-22}$. Por su parte, el BIA es una entidad cuya prevalencia aumenta con la edad, motivo por el que la edad constituye un factor de confusión positivo a la hora de estimar la asociación entre FA postoperatoria y BIA. Por ello, decidimos explorar esta misma asociación en sujetos más jóvenes, evidenciando que la presencia de BIA-AV, aun siendo una entidad menos frecuente en pacientes jóvenes, se asocia de forma independiente con la aparición de FA tras la cirugía cardiaca. 


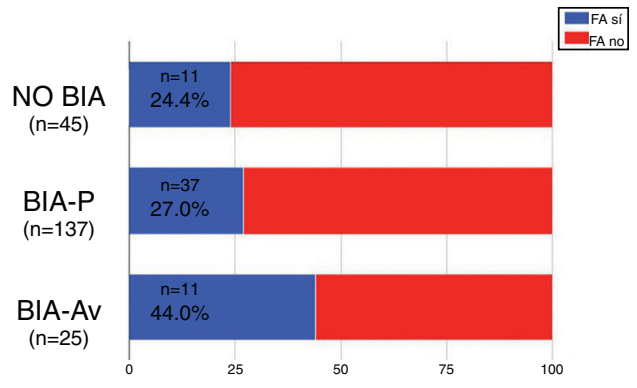

Figura 3 Se muestra la frecuencia de fibrilación auricular (FA) postoperatoria en función de la presencia de bloqueo interauricular parcial (BIA-P) o avanzado (BIA-AV) en comparación con la ausencia de BIA (NO BIA).

Tabla 2 Variables asociadas de forma independiente y estadísticamente significativa con FA postoperatoria

\begin{tabular}{lll}
\hline & OR (IC 95\%) & $\mathrm{p}$ \\
\hline Dilatación AI & $5.02(1.75-14.45)$ & 0.003 \\
Edad & $1.08(1.01-1.15)$ & 0.025 \\
BIA avanzado & $4.50(1.01-20.15)$ & 0.049 \\
\hline
\end{tabular}

Al: aurícula izquierda; BIA: bloqueo interauricular; FA: fibrilación auricular; IC: intervalo de confianza; OR: odds ratio.

El síndrome de Bayés ha sido ampliamente estudiado en múltiples escenarios, incluyendo la cardioversión farmacológica de $\mathrm{FA}^{23}$, la ablación de venas pulmonares ${ }^{24}$ o la ablación del istmo cavotricuspídeo ${ }^{25}$, confirmando en todos ellos una asociación entre la presencia de BIA y la recurrencia o nueva aparición de FA. Se ha postulado también que la presencia de BIA constituye un factor de riesgo para presentar FA en pacientes con miocardiopatía hipertrófica ${ }^{26} 0$ con miocardiopatía por enfermedad de $\mathrm{Chagas}^{27}$. Se ha sugerido también que la mayor prevalencia de BIA existente en pacientes con síndrome de apnea obstructiva del sueño podría justificar en cierta medida la mayor frecuencia de taquiarritmias auriculares en este grupo de pacientes ${ }^{28}$.

En el año 2014, Conde et al. ${ }^{29}$ investigaron la asociación de BIA y FA tras cirugía cardiaca. En este estudio, en el que se incluyeron un total de 277 pacientes sometidos a cirugía de revascularización coronaria (edad media de $64.8 \pm 10.3$ ), la proporción de FA postoperatoria fue del $37.9 \%$, sin encontrarse diferencias significativas entre el grupo de pacientes con BIA y el grupo de pacientes sin BIA (35.2\% vs. $39.4 \%$; $p=0.504)$. Nuestro estudio, en cambio, incluyó pacientes sometidos a distintos tipos de cirugía cardiaca, con una proporción de FA postoperatoria algo menor, dato que podría justificarse por una menor edad media de nuestra población. Otra de las diferencias que se deben resaltar es la frecuencia BIA-P (3 veces mayor en nuestro estudio), si bien la frecuencia de BIA-AV fue similar. Con todo ello, la proporción de FA postoperatoria fue muy similar en pacientes sin BIA, con BIA-P y con BIA-AV en el estudio de Conde et al., mientras que en nuestro estudio la aparición de FA en pacientes sin BIA y con BIA-P fue comparable en ambos grupos y menor que en los mismos pacientes del mencionado estudio. Por otra parte, la presencia de BIA-AV se asoció claramente con una mayor proporción de FA postoperatoria en nuestros
Tabla 3 Resultados comparativos entre estudios

\begin{tabular}{lll}
\hline & $\begin{array}{l}\text { Conde et al. }{ }^{29} \\
(\mathrm{n}=277)\end{array}$ & $\begin{array}{l}\text { Nuestro estudio } \\
(\mathrm{n}=207)\end{array}$ \\
\hline Edad & $64.8 \pm 10.3$ & $53.1 \pm 9.8$ \\
FA postoperatoria & $37.9 \%$ & $28.5 \%$ \\
BIA-P & $21.5 \%$ & $66.2 \%$ \\
BIA-AV & $14.1 \%$ & $12.1 \%$ \\
FA en NO BIA & $39.4 \%$ & $24.4 \%$ \\
FA en BIA-P & $36.4 \%$ & $27.0 \%$ \\
FA en BIA-AV & $33.3 \%$ & $44.0 \%$ \\
\hline
\end{tabular}

BIA-AV: bloqueo interauricular avanzado; BIA-P: bloqueo interauricular parcial; FA: fibrilación auricular; NO BIA: ausencia de bloqueo interauricular.

pacientes, hallazgo no objetivado en el estudio previamente citado. En la tabla 3 se muestran de forma comparativa los principales datos de ambos estudios.

Uno de los puntos a discutir es la elevada prevalencia de BIA objetivada en nuestra muestra $(78.3 \%$ del total de pacientes incluyendo BIA-P y BIA-AV). Según estudios observacionales previos llevados a cabo en población hospitalaria, un $47 \%$ de sujetos ingresados presentaban BIA, siendo esta prevalencia mayor en sujetos de más de 50 años (en torno a un $50-60 \%$ ) y menor en sujetos jóvenes (10-20\% en sujetos de entre 20 y 40 años de edad) $)^{30}$. Se sabe además que la prevalencia de su forma avanzada es prácticamente inexistente (menor del 1\%) en población joven, mientras que en pacientes septuagenarios y centenarios en ritmo sinusal se han documentado frecuencias del 9 y $35 \%$, respectivamente ${ }^{31}$. Por tanto, el aumento de prevalencia de BIA con la edad es un hecho constatado. Con todo ello, teniendo en cuenta que la edad media de nuestra muestra se encuentra entre los 50 y 60 años, y recalcando que la inmensa mayoría de estos pacientes presentan cardiopatía significativa de base, es esperable una prevalencia de BIA mayor que la reportada en otros estudios. Sin embargo, volviendo de nuevo a la comparación con el estudio de Conde et al., donde la frecuencia de BIA-P fue mucho menor que en nuestra población a pesar de contar con una mayor edad media, debe otorgarse un mayor peso a la afectación estructural cardiaca para poder explicar esta diferencia.

Es habitual la coexistencia de dilatación auricular y BIA. El aumento de la duración de la onda P sobre el ECG de superficie en pacientes con crecimiento auricular es consecuencia tanto de la presencia de BIA asociado como del aumento que se produce en la distancia que tiene que recorrer el impulso eléctrico para despolarizar ambas aurículas, teniendo probablemente este mecanismo menor relevancia que el primero ${ }^{32}$. Sin embargo, considerando que el BIA puede aparecer en pacientes sin dilatación auricular, que puede ser además un fenómeno transitorio y que se puede reproducir de forma experimental, es adecuado afirmar que se trata de una entidad diferenciada ${ }^{33}$. En nuestro estudio, aunque se observó una clara asociación entre la dilatación auricular y la presencia de BIA-AV, un $8.8 \%$ de los pacientes sin crecimiento significativo auricular presentaban BIA-AV, dato que apoya que la dilatación auricular y el trastorno en la conducción auricular constituyen entidades diferenciadas que con frecuencia coexisten. 
En nuestros resultados, encontramos también una asociación entre los niveles de NTproBNP y la aparición de FA. Si bien no resultó asociarse de forma independiente con FA postoperatoria, este dato ya ha sido reflejado en la literatura: se trata de un parámetro que ha demostrado ser marcador independiente de la aparición de este tipo de arritmia en el postoperatorio de cirugía cardiaca ${ }^{34}$.

La FA postoperatoria se ha asociado a múltiples complicaciones, entre las que se incluye el ictus tras la cirugía; se ha relacionado también con una mayor mortalidad tanto a corto como a largo plazo e implica también un mayor tiempo de ingreso hospitalario ${ }^{13,35-39}$. Existen estudios que han demostrado una elevada incidencia de FA en el seguimiento posterior, con un riesgo de presentar episodios subsecuentes de FA que puede llegar a ser 8 veces mayor en comparación con pacientes que no tuvieron FA postoperatoria ${ }^{40,41}$. La incidencia de episodios de FA en el seguimiento a largo plazo de estos pacientes es aún mayor (algo más del $40 \%$ a 2 años) cuando se utilizan dispositivos de monitorización continua (Holter insertable) ${ }^{42}$. Por todo ello, es fundamental identificar a aquellos pacientes con un mayor riesgo de presentar esta arritmia postoperatoria e implementar las medidas preventivas necesarias de acuerdo con las guías de práctica clínica. Serán necesarios estudios para comprobar si la presencia de BIA supone un riesgo adicional para presentar nuevos episodios de FA y otras complicaciones asociadas en el seguimiento a largo plazo y para conocer el beneficio de las distintas estrategias que se utilizan en la prevención de esta arritmia en este grupo de pacientes.

\section{Limitaciones}

Se trata de un estudio unicéntrico, hecho que puede limitar la validez externa de los resultados. Solo se reflejaron aquellos episodios de FA que acontecieron durante el ingreso sin haber registrado los que se pudieron haber producido tras el alta hospitalaria, por lo que es posible que la incidencia de FA postoperatoria esté infraestimada. No se ha recogido información acerca de la utilización de catecolaminas en el postoperatorio ni de fármacos que puedan reducir el riesgo de FA (betabloqueantes, fármacos antiarrítmicos).

\section{Conclusiones}

El BIA es una entidad frecuente en pacientes sometidos a cirugía cardiaca y de fácil diagnóstico mediante ECG de superficie. En su forma avanzada, se asocia de forma independiente con la aparición de FA después de la cirugía cardiaca en pacientes menores de 65 años.

\section{Responsabilidades éticas}

Protección de personas y animales. Los autores declaran que para esta investigación no se han realizado experimentos en seres humanos ni en animales.

Confidencialidad de los datos. Los autores declaran que han seguido los protocolos de su centro de trabajo sobre la publicación de datos de pacientes.

\section{Financiación}

Ninguna.

\section{Conflicto de intereses}

Los autores declaran no tener ningún conflicto de intereses.

\section{Bibliografía}

1. Bachmann G. The inter-auricular time interval. Am J Physiol. 1916;41:309-20.

2. Bachmann G. The significance of splitting of the p-wave in the electrocardiogram. Ann Intern Med. 1941;14:1702.

3. Bayés de Luna A. Bloqueo a nivel auricular. Rev Esp Cardiol. 1979;32:5-10.

4. Bayés de Luna A, Cladellas M, Oter R, et al. Interatrial conduction block and retrograde activation of the left atrium and paroxysmal supraventricular tachyarrhythmia. Eur Heart J. 1988;9:1112-8

5. Conde D, Baranchuk A. Interatrial block as anatomical-electrical substrate for supraventricular arrhythmias: Bayés syndrome. Arch Cardiol Mex. 2014;84:32-40.

6. Conde D, Baranchuk A. Síndrome de Bayés: lo que un cardiólogo no debe dejar de saber. Rev Argent Cardiol. 2014;82:237-9.

7. Lorbar M, Levrault R, Phadke JG, et al. Interatrial block as a predictor of embolic stroke. Am J Cardiol. 2005;95:667-8.

8. Ariyarajah V, Apiyasawat S, Najjar H, et al. Frequency of interatrial block in patients with sinus rhythm hospitalized for stroke and comparison to those without interatrial block. Am J Cardiol. 2007;99:49-52.

9. Kamel H, Hunter M, Moon YP, et al. Electrocardiographic left atrial abnormality and risk of stroke. Stroke. 2015;46:3208-12.

10. Nielsen JB, Kühl JT, Pietersen A, et al. P-wave duration and the risk of atrial fibrillation: Results from the Copenhagen ECG Study. Heart Rhythm. 2015;12:1887-95.

11. Martinez-Selles M, García-Izquierdo E, Lozano IF. Anticoagulation in elderly patients at high risk of atrial fibrillation without documented arrhythmias. J Geriatr Cardiol. 2017;14:166-8.

12. Maisel WH, Rawn JD, Stevenson WG. Atrial fibrillation after cardiac surgery. Ann Intern Med. 2001;135:1061-73.

13. Creswell LL, Schuessler RB, Rosenbloom M, et al. Hazards of postoperative atrial arrhythmias. Ann Thorac Surg. 1993;56:539-49.

14. Maesen B, Nijs J, Maessen J, et al. Post-operative atrial fibrillation: A maze of mechanisms. Europace. 2012;14:159-74.

15. Caretta Q, Mercanti CA, de Nardo D, et al. Ventricular conduction defects and atrial fibrillation after coronary artery bypass grafting. Multivariate analysis of preoperative, intraoperative and postoperative variables. Eur Heart J. 1991;12:1107-11.

16. Mendes LA, Connelly GP, McKenney PA, et al. Right coronary artery stenosis: An independent predictor of atrial fibrillation after coronary artery bypass surgery. J Am Coll Cardiol. 1995;25:198-202.

17. Aranki SF, Shaw DP, Adams DH, et al. Predictors of atrial fibrillation after coronary artery surgery. Current trends and impact on hospital resources. Circulation. 1996;94:390-7.

18. Asher CR, Miller DP, Grimm RA, et al. Analysis of risk factors for development of atrial fibrillation early after cardiac valvular surgery. Am J Cardiol. 1998;82:892-5.

19. Zaman AG, Archbold RA, Helft G, et al. Atrial fibrillation after coronary artery bypass surgery: A model for preoperative risk stratification. Circulation. 2000;101:1403-8.

20. Mathew JP, Fontes ML, Tudor IC, et al. A multicenter risk index for atrial fibrillation after cardiac surgery. JAMA. 2004;291:1720-9. 
21. Zacharias A, Schwann TA, Riordan CJ, et al. Obesity and risk of new-onset atrial fibrillation after cardiac surgery. Circulation. 2005;112:3247-55.

22. Shirzad M, Karimi A, Tazik M, et al. Factores determinantes de fibrilación auricular postoperatoria y el uso de recursos en cirugía cardiaca. Rev Esp Cardiol. 2010;63:1054-60.

23. Enriquez A, Conde D, Hopman W, et al. Advanced interatrial block is associated with recurrence of atrial fibrillation post pharmacological cardioversion. Cardiovasc Ther. 2014;32:52-6.

24. Wu J-T, Long D-Y, Dong JZ, et al. Advanced interatrial block predicts clinical recurrence of atrial fibrillation after catheter ablation. J Cardiol. 2016;68:352-6.

25. Enriquez A, Sarrias A, Villuendas R, et al. New-onset atrial fibrillation after cavotricuspid isthmus ablation: Identification of advanced interatrial block is key. Europace. 2015;17:1289-93.

26. Ozdemir O, Soylu M, Demir AD, et al. P-wave durations as a predictor for atrial fibrillation development in patients with hypertrophic cardiomyopathy. Int J Cardiol. 2004;94:163-6.

27. Enriquez A, Conde D, Femenia F, et al. Relation of interatrial block to new-onset atrial fibrillation in patients with Chagas cardiomyopathy and implantable cardioverter-defibrillators. Am J Cardiol. 2014;113:1740-3.

28. Baranchuk A, Parfrey B, Lim L, et al. Interatrial block in patients with obstructive sleep apnea. Cardiol J. 2011;18:171-5.

29. Conde D, van Oosten EM, Hamilton A, et al. Prevalence of interatrial block in patients undergoing coronary bypass graft surgery. Int J Cardiol. 2014;171:e98-9.

30. Asad N, Spodick DH. Prevalence of interatrial block in a general hospital population. Am J Cardiol. 2003;91:609-10.

31. Russo V, Marano M. Letter to the Editor. Prevalence of interatrial block during lifetime. Heart Rhythm. 2016;13:e90-1.

32. Chhabra L, Devadoss R, Chaubey VK, et al. Interatrial block in the modern era. Curr Cardiol Rev. 2014;10:181-9.

33. Bayés de Luna A, Platonov P, Cosio FG, et al. Interatrial blocks. A separate entity from left atrial enlargement: A consensus report. J Electrocardiol. 2012;45:445-51.
34. Wazni OM, Martin DO, Marrouche NF, et al. Plasma B-type natriuretic peptide levels predict postoperative atrial fibrillation in patients undergoing cardiac surgery. Circulation. 2004;110:124-7.

35. Lahtinen J, Biancari F, Salmela E, et al. Postoperative atrial fibrillation is a major cause of stroke after on-pump coronary artery bypass surgery. Ann Thorac Surg. 2004;77:1241-4.

36. Villareal RP, Hariharan R, Liu BC, et al. Postoperative atrial fibrillation and mortality after coronary artery bypass surgery. J Am Coll Cardiol. 2004;43:742-8.

37. Mariscalco G, Klersy C, Zanobini M, et al. Atrial fibrillation after isolated coronary surgery affects late survival. Circulation. 2008;118:1612-8.

38. Bramer S, van Straten AHM, Soliman Hamad MA, et al. The impact of new-onset postoperative atrial fibrillation on mortality after coronary artery bypass grafting. Ann Thorac Surg. 2010;90:443-9.

39. Saxena A, Dinh DT, Smith JA, et al. Usefulness of postoperative atrial fibrillation as an independent predictor for worse early and late outcomes after isolated coronary artery bypass grafting (multicenter Australian study of 19,497 patients). Am J Cardiol. 2012;109:219-25.

40. Ahlsson A, Fengsrud E, Bodin L, et al. Postoperative atrial fibrillation in patients undergoing aortocoronary bypass surgery carries an eightfold risk of future atrial fibrillation and a doubled cardiovascular mortality. Eur J Cardiothorac Surg. 2010;37:1353-9.

41. Pillarisetti J, Patel A, Bommana S, et al. Atrial fibrillation following open heart surgery: Long-term incidence and prognosis. J Interv Card Electrophysiol. 2014;39:69-75.

42. El-Chami MF, Merchant FM, Smith P, et al. Management of new-onset postoperative atrial fibrillation utilizing insertable cardiac monitor technology to observe recurrence of AF (MONITOR-AF). Pacing Clin Electrophysiol. 2016;39: 1083-9. 